\title{
Constraints on barotropic dark energy models by a new phenomenological $q(z)$ parameterization
}

\author{
Jaime Román-Garza ${ }^{1,2, a} \oplus$, Tomás Verdugo ${ }^{2}$, Juan Magaña ${ }^{3}$, Verónica Motta ${ }^{3}$ \\ ${ }^{1}$ Facultad de Ciencias Físico Matemáticas, Universidad Autónoma de Nuevo León, San Nicolás de los Garza, Mexico \\ ${ }^{2}$ Instituto de Astronomía, Universidad Nacional Autónoma de México, Apdo. postal 106, CP 22800 Ensenada, BC, Mexico \\ ${ }^{3}$ Instituto de Física y Astronomía, Universidad de Valparaíso, Avenida Gran Bretaña, 1111 Valparaiso, Chile
}

Received: 5 July 2019 / Accepted: 9 October 2019 / Published online: 5 November 2019

(C) The Author(s) 2019

\begin{abstract}
In this paper, we propose a new phenomenological two parameter parameterization of $q(z)$ to constrain barotropic dark energy models by considering a spatially flat Universe, neglecting the radiation component, and reconstructing the effective equation of state (EoS). This two free-parameter EoS reconstruction shows a non-monotonic behavior, pointing to a more general fitting for the scalar field models, like thawing and freezing models. We constrain the $q(z)$ free parameters using the observational data of the Hubble parameter obtained from cosmic chronometers, the joint-light-analysis Type Ia Supernovae (SNIa) sample, the Pantheon (SNIa) sample, and a joint analysis from these data. We obtain, for the joint analysis with the Pantheon (SNIa) sample a value of $q(z)$ today, $q_{0}=-0.51_{-0.10}^{+0.09}$, and a transition redshift, $z_{t}=0.65_{-0.17}^{+0.19}$ (when the Universe change from an decelerated phase to an accelerated one). The effective EoS reconstruction and the $\omega^{\prime}-\omega$ plane analysis point towards a transition over the phantom divide, i.e. $\omega=-1$, which is consistent with a non parametric EoS reconstruction reported by other authors.
\end{abstract}

\section{Introduction}

Several cosmological observations indicate that the Universe experiments a late-time acceleration [1]. This feature was evidenced for the first time by the observations of distant Type Ia Supernovae (SNIa) [2,3] and is one of the major puzzles in modern cosmology. In general, there are two ways to explain this mysterious cosmic phase: i) to postulate a fluid with negative pressure, the so-called dark energy (DE), into the canonical Einstein's general relativity theory or ii) to modify the gravity laws. Between these two approaches, numerous models have been proposed. Most of them can explain

\footnotetext{
a e-mail: jaime.romangrz@uanl.edu.mx
}

a wide range of the cosmological observations and distinguishing among them is not a trivial problem. Despite of this, one simple model has been established as the standard, the one with a cosmological constant associated to the quantum vacuum fluctuations $\Lambda$ with cold dark matter $(\Lambda \mathrm{CDM})$. Nevertheless, it has theoretical problems [4,5] which motivates further studies of alternative models [6]. For instance, some of those consider a dynamical DE involving scalar fields, like quintessence [7-9], phantom [10,11], quintom [12], and $\mathrm{k}$-essence fields $[13,14]$. An advantage of these models is that the DE equation of state (EoS) evolves with time, and thus it can be parameterized by a function of the scale factor (redshift, as proposed by $[15,16]$ ) to explore its cosmological behavior.

The standard way to examine these models is to calculate the Friedmann and Raychaudhuri equations in a background cosmology to constrain their free parameters (see for example [17]). A model-independent approach is to investigate the cosmographic parameters that characterize the kinematics of the cosmic expansion [18-23]. The advantage of this procedure is that the only assumption is the Cosmological Principle, i.e. an homogeneous and isotropic Universe, without speculating about its composition. Indeed, it is very common to consider the Hubble parameter, $H \equiv \dot{a} / a$, and the deceleration parameter, $q(a) \equiv-\ddot{a} a / \dot{a}^{2} .{ }^{1}$ However, higher order derivatives of the scale factor $a$, such as jerk and snap, can be also considered, e.g. [25]. By probing the cosmographic parameters using cosmological data, we can associate them to a given dynamical DE entity and reconstruct its features as well as the Universe dynamics. In this vein, several authors have proposed a number of functions to parameterize the deceleration parameter $q(z)$ (see for example [25-30] for recent studies) and associate its features to a some DE model.

\footnotetext{
1 Alan Sandage claimed that the cosmic expansion can be determined by these two parameters at $\mathrm{z}=0$ [24].
} 
The motivation of the present work is to propose a new parameterization of the deceleration parameter as function of redshift, based only in the cosmological principle, and able to generate an EoS which describes both, slowly and rapidly behaviors [31]. The ansatz is a continuous and differentiable function that is valid from the matter domination epoch until the near future. We constrain the $q(z)$ free parameter by performing a Bayesian analysis for which we employ the latest compilation of observational Hubble data (hereinafter OHD) from cosmic chronometers and Type Ia Supernova. Using the mean value parameters, we reconstruct an effective EoS to the dynamical dark energy.

The paper is organized as follows. In Sect. 2 we state the theoretical framework and present the parametric equation of the deceleration parameter. Section 3 provides a description of the data sets and the methodology used to constrain the parameters of the deceleration parameter. Section 4 presents the obtained EoS, and the tools to discriminate between different DE models. Finally, in Sect. 5 the remarks and conclusions are presented.

\section{Theoretical framework}

2.1 Proposed parameterization for the deceleration parameter

The deceleration parameter as function of $H(z)$ is

$q=-\left(1+\frac{\dot{H}}{H^{2}}\right)$,

if $q>0$ the Universe is at a decelerated phase, otherwise $q<0$ corresponds to an accelerated phase. By integrating the Eq. (1), the Hubble parameter can be written as:

$H(z)=H_{0} \exp \left(\int_{0}^{z} \frac{1+q\left(z^{\prime}\right)}{1+z^{\prime}} d z^{\prime}\right)$,

where $H_{0}$ is the Hubble parameter at the present epoch and $z=(1 / a)-1$ is the redshift.

The OHD suggest that $q<0$ at the present epoch and $q>0$ during an early epoch when the matter dominates as shown in Refs. [32,33]. The structure formation at this early epoch is explained by a decelerated phase, so the value of the deceleration parameter transit from positive in the past to negative at the present. The parameterization of the deceleration parameter is a useful method to reconstruct cosmological parameters and constrain the dynamical evolution of the universe in a general scheme [34]. There are several parameterizations for $q(z)$ reported in the literature, see Refs. [2530,32,34-39]. We propose a new one as follows

$q(z)=q_{1}+\left(q_{0}-q_{1}\right)(z+1) e^{z_{c}^{2}-\left(z+z_{c}\right)^{2}}$, where, $q_{0}$ and $q_{1}$ are the values for the deceleration parameter at the present epoch, and at high redshift, respectively. We set $q_{1}=0.5$ to consider the matter-dominated epoch of the Universe. The characteristic redshift, $z_{c}$, is a free parameter related to the transition redshift, $z_{t}$, the redshift at which the Universe underwent a transition from deceleration to an acceleration phase. This is a well behaved parameterization (see Fig. 1) that can reproduce a soft step transition, as well as changes in concavity in the deceleration parameter (notice that both an accelerated and decelerated stage at $z=0$ are allowed), and facilitates the analytical reconstruction of other cosmological parameters like $H(z)$, and $w(z)$. Note how combinations of $q_{0}$ and $z_{c}$ can yield the same transition redshift.

Substituting the Eq. (3) into the Eq. (2), we obtain the analytical expression for the Hubble parameter in terms of $z$ :

$H(z)=H_{0}(z+1)^{q_{1}+1} e^{\xi \eta}$,

where $\xi=(\sqrt{\pi} / 2)\left(q_{0}-q_{1}\right) e^{z_{c}^{2}}, \eta=\operatorname{erf}\left(z+z_{c}\right)-\operatorname{erf}\left(z_{c}\right)$, and $\operatorname{erf}(x)$ is the error function of $x$. This is the expression that is fitted to the data.

\subsection{The effective equation of state}

With the metric for a spatially flat Friedmann-LemaîtreRobertson-Walker (FLRW) space-time,

$d s^{2}=-d t^{2}+a^{2}(t)\left\{d r^{2}+r^{2} d \Omega^{2}\right\}$

and considering a space-time composed of a non-relativistic component $\rho_{m}$ and a barotropic fluid with an effective density $\rho_{\text {eff }}$ and an effective pressure $p_{\text {eff }}$, the Einstein field equations in units of $8 \pi G=c=1$ are obtained following Ref. [4] as

$$
\begin{aligned}
3 H^{2} & =\rho_{m}+\rho_{\mathrm{eff}}, \\
2 \dot{H}+3 H^{2} & =-p_{\text {eff }}
\end{aligned}
$$

and the effective EoS is written as

$\omega=\frac{p_{\mathrm{eff}}}{\rho_{\mathrm{eff}}}$.

Substituting (6) and (7) in (8), the EoS in terms of $q(z)$ and $H(z)$ is obtained following Ref. [30] as

$\omega(z)=\frac{2}{3} \frac{q(z)-\frac{1}{2}}{1-\Omega_{m, 0}(1+z)^{3}\left(\frac{H_{0}}{H(z)}\right)^{2}}$.

where $\Omega_{m, 0}$ is the matter density parameter $\Omega_{m}=\rho_{m} / \rho_{\text {crit }}$ evaluated at $z=0 .^{2}$

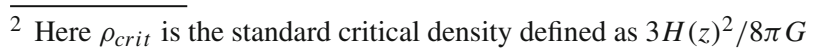



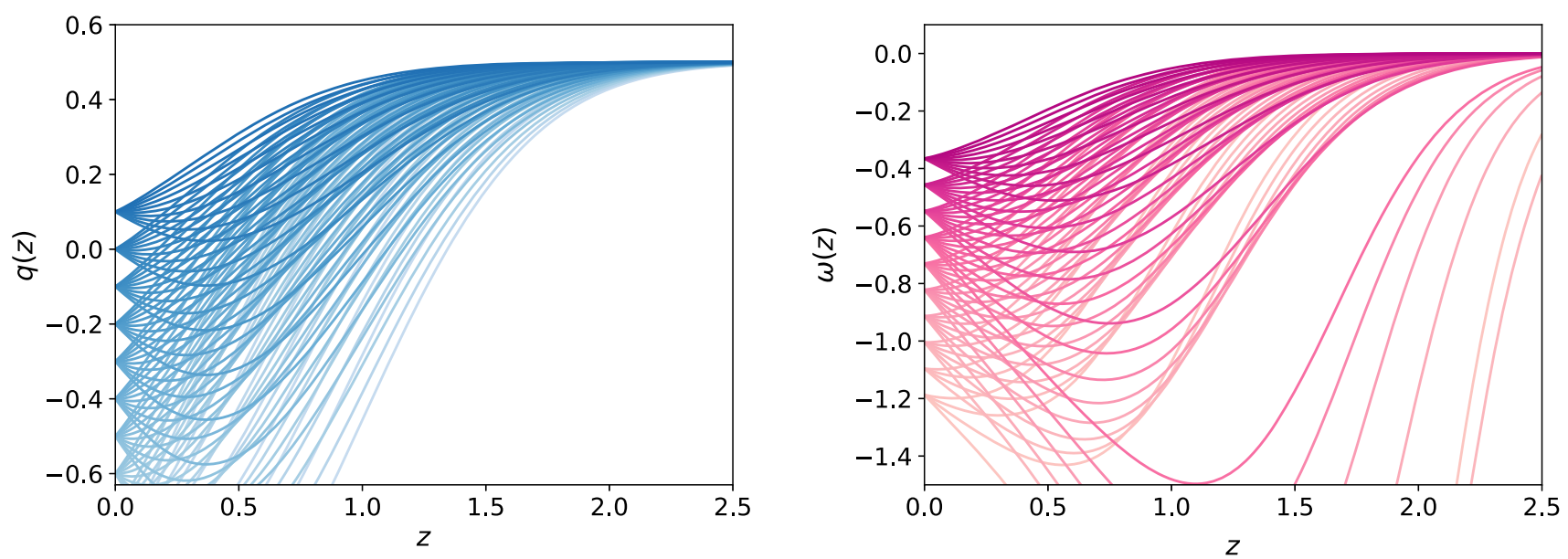

Fig. 1 Left panel: functional form of the proposed $q(z)$ given by Eq. (3) for different $\left(q_{0}, z_{c}\right)$ values. Notice that both an accelerated and decelerated stage at $z=0$ are allowed. Right panel: functional form of $\omega(z)$ calculated through Eq. (9)

By substituting Eqs. (3) and (4) in Eq. (9), we obtain the expression

$\omega(z)=\frac{2}{3} \frac{\left(q_{0}-q_{1}\right)(z+1) \exp \left(z_{c}^{2}-\left(z+z_{c}\right)^{2}\right)}{1-\Omega_{m, 0}(1+z)^{1-q_{1}} \exp (-2 \xi \eta)}$.

The right panel of Fig. 1 shows how the EoS changes for different values of $q_{0}$ and $z_{c}$. The reconstruction of $\omega(z)$ yield distinct DE behaviors when the barotropic fluid is associated to a minimally coupled scalar field: quintessence $(-1 \leq \omega(z) \leq 1)$, phantom $(\omega(z)<-1)$ or even crossing the phantom divide, $\omega=-1$, e.g. quintom models (where the DE component moves across the quintessence and phantom regions through two scalar fields) see [4] and references therein. In contrast to some $\omega(z)$ parameterizations analyzed in the literature [15-17,40-44], our EoS concavity changes from low to high $z$ values if there is at least one inflexion point at $z>0$. Some authors have proposed a more general form for the EoS parameterization, with a different approach in which a transition function introduces a rapid evolution of $w(z)[31,45,46]$. In the present work, we obtain a similar result, however the main difference is that the behavior of the EoS is a direct result of the proposed $q(z)$ parameterization. Indeed, this further highlights the importance of the proposed functional form for the deceleration parameter.

\section{Observational data and methodology}

In this section we introduce the cosmological data and the methodology used to constraint the $q(z)$ free parameters of the Eq. (3).

\subsection{Observational Hubble data from cosmic chronometers}

Several authors have shown that the OHD can be used to constrain cosmological parameters. There are two techniques to measure the cosmic expansion at different redshifts: using the baryon acoustic oscillation analysis or applying the differential age technique (DA) in cosmic chronometers, i.e. passive-early-type galaxies. This last method was proposed by [47] and measures $H(z)$ using the following relation for two early-type galaxies separated by a small redshift interval $\Delta z$

$H(z)=-\frac{1}{1+z} \frac{\mathrm{dz}}{\mathrm{dt}}$,

where $\mathrm{dz} / \mathrm{dt}$ is measured by estimating the differential age $\Delta t$ with the $4000 \AA$ break $(D 4000)$ feature in their spectra.

We employ the latest OHD obtained from DA in cosmic chronometers, which contains 31 data points covering $0<z<1.97$, compiled by [48] and references therein. The figure-of-merit for the OHD is written as

$\chi_{\mathrm{OHD}}^{2}=\sum_{i=1}^{31} \frac{\left[H\left(z_{i}\right)-H_{D A}\left(z_{i}\right)\right]^{2}}{\sigma_{H_{i}}^{2}}$,

where $H\left(z_{i}\right)$ is the theoretical Hubble parameter, $H_{D A}\left(z_{i}\right)$ is the observational one at redshift $z_{i}$, and $\sigma_{H_{i}}$ is its uncertainty.

\subsection{Type Ia Supernovae}

The standard test to investigate the accelerating expansion is employing the observations of type SNIa at high redshifts. We use two of the latest SNIa compilations, the so-called joint-light-curve-analysis (JLA) [49] sample, that contains 740 points spanning a redshift range $0.01<z<1.2$, and the Pantheon sample [50] containing 1048 points in the redshift range $0.001<z<2.3$. 


\subsubsection{JLA SNIa sample}

The figure-of-merit for the JLA data is given by

$$
\chi_{\mathrm{JLA}}^{2}=\left(\hat{\mu}_{\mathrm{JLA}}-\mu_{q z}\right)^{\dagger} \mathrm{C}_{\eta}^{-1}\left(\hat{\mu}_{\mathrm{JLA}}-\mu_{q z}\right) \text {, }
$$

where $\mu_{q z}=5 \log _{10}\left(d_{L} / 10 \mathrm{pc}\right)$ is the theoretical distance modulus for the $q(z)$ parameterization and $d_{L}$ is the luminosity distance given by

$d_{L}=c(1+z) \int_{0}^{z} \frac{\mathrm{dz}^{\prime}}{H\left(z^{\prime}\right)}$.

The observational distance modulus, $\hat{\mu}$, for the the JLA data reads as

$\hat{\mu}_{\mathrm{JLA}}=m_{b}^{\star}-\left(M_{B}-\alpha \times X_{1}+\beta \times C\right)$,

where $m_{b}^{\star}$ corresponds to the observed peak magnitude, $M_{B}$ is the $B$-band absolute magnitude. The $X_{1}$ and $C$ variables describe the time stretching of the light-curve and the Supernova color at maximum brightness respectively. The $\alpha$, and $\beta$ coefficients are nuisance parameters. For JLA sample, the absolute magnitude $M_{B}$ is related to the host stellar mass, $M_{\text {stellar }}$ by the step function:

$M_{B}= \begin{cases}M_{B}^{1} & \text { if } \mathrm{M}_{\text {stellar }}<10^{10} \mathrm{M}_{\odot} \\ M_{B}^{1}+\Delta_{M} & \text { otherwise. }\end{cases}$

Finally, $C_{\eta}$ is the covariance matrix ${ }^{3}$ of $\hat{\mu}$ provided by [49], which takes into account several statistical and systematic errors in the SNIa data.

\subsubsection{Pantheon SNIa sample}

The observational distance modulus $\mu_{\mathrm{PAN}}$ for Pantheon SNIa can be measured as

$\mu_{\mathrm{PAN}}=m_{b}^{\star}-M_{B}+\alpha \times X_{1}-\beta \times C+\Delta_{M}+\Delta_{B}$,

where the parameters $m_{b}^{\star}, M_{B}, \alpha, X_{1}, \beta$, and $C$ are the same as the JLA sample. $\Delta_{M}$ is a distance correction based on the host-galaxy mass of the SNIa and $\Delta_{B}$ is a distance correction based on predicted biases from simulations. It is worthy to note that [50] provided $\mu_{\mathrm{PAN}}=\mu_{\mathrm{PAN}}+M_{B}$, thus, we can marginalize over the $M_{B}$ parameter. The marginalized figureof-merit for the Pantheon sample is given by

$\chi_{\text {Pan }_{\text {M marg }}}^{2}=a+\log \left(\frac{e}{2 \pi}\right)-\frac{b^{2}}{e}$,

where $a=\Delta \tilde{\boldsymbol{\mu}}^{T} \cdot \mathbf{C}_{\mathbf{P}}^{-\mathbf{1}} \cdot \Delta \tilde{\boldsymbol{\mu}}, b=\Delta \tilde{\boldsymbol{\mu}}^{T} \cdot \mathbf{C}_{\mathbf{P}}^{-\mathbf{1}} \cdot \Delta \mathbf{1}, e=\Delta \mathbf{1}^{T}$. $\mathbf{C}_{\mathbf{P}}^{-\mathbf{1}} \cdot \Delta \mathbf{1}$, and $\Delta \tilde{\boldsymbol{\mu}}$ is the vector of residuals between the model distance modulus and the observed $\mu_{\mathrm{PAN}}$. The covariance matrix $\mathbf{C}_{\mathbf{P}}$ can be constructed as $\mathbf{C}_{\mathbf{P}}=\mathbf{C}_{\mathbf{P}, \text { stat }}+\mathbf{C}_{\mathbf{P}, \mathbf{s y s}}$,

\footnotetext{
3 Available at http://supernovae.in2p3.fr/sdss_snls_jla/ReadMe.html.
}

where $\mathbf{C}_{\mathbf{P} \text {,sys }}$ is the systematic covariance matrix, and $\mathbf{C}_{\mathbf{P} \text {,stat }}$ is a diagonal matrix which contains the statistical errors on $\mu_{\text {PAN }}$. We refer the interested reader to [50] for a detailed description how these matrices are constructed.

\subsection{Fitting the data}

To estimate the values of the parameters $q_{0}$ and $z_{c}$ from Eq. (3), a Markov Chain Monte Carlo (MCMC) Bayesian statistical analysis is performed using the Affine-Invariant MCMC Ensemble sampler from the emcee Python module [51]. We perform the following cases: using only the CC data, only a SNIa sample (JLA or Pantheon), and the joint analysis $\mathrm{CC}+\mathrm{SNIa}$ (JLA or Pantheon).The computations are running with 1500 steps to stabilize the estimations (burn-in phase), and $5000 \mathrm{MCMC}$ steps using 600 walkers. We assume the following flat priors for all cases: $h \in[0,1], q_{0} \in[-1,1]$, $z_{c} \in[0,2]$. When the JLA sample is used in the analysis, we also consider $M_{b}^{1} \in[-20.0,-18.0], \Delta_{M} \in[-0.1,0.1]$, $\alpha \in[0.0,0.2]$, and $\beta \in[0.0,4.0]$. To assess the convergence of our analysis, a Gelman-Rubin test is employed.

We assume a Gaussian likelihood when the parameter estimation is performed using only a data set. The goodness of the fit for the joint analysis is quantified by a total $\chi^{2}$ defined as:

$\chi_{T}^{2}=\chi_{\mathrm{OHD}}^{2}+\chi_{\mathrm{SNIa}}^{2}$,

where $\chi_{\mathrm{OHD}}^{2}$ is calculated using Eq. (12). And $\chi_{\mathrm{SNIa}}^{2}$ is calculated using Eq. (13) or (18) for the JLA or Pantheon sample respectively. Thus, a joint Gaussian likelihood can be expressed as:

$\mathcal{L}_{\text {joint }} \propto \exp \left(-\chi_{T}^{2} / 2\right)$,

where $\mathcal{L}_{\text {joint }}$ is the product of the likelihood functions of each data set.

The mean values of the fits are presented in Table 1. Figure 2 shows the confidence contours obtained for the joint analysis, for both, the JLA and the Pantheon samples. In the left panel of Fig. 3 we show the OHD along with the function given by Eq. (4) using the mean values obtained from the joint analysis ( $\mathrm{CC}+$ Pantheon) fitting. In the right panel of the same figure is the reconstructed deceleration parameter with these same constraints.

Along with the narrow constraints obtained with the joint analysis (see Fig. 2), we note an anticorrelation between $z_{c}$ and $q_{0}$ parameters. This degeneracy has a mathematical origin: as $q_{0}$ becomes less negative, the transition redshift $z_{t}$ is larger, ${ }^{4}$ which in turn decreases $z_{c}$ (see Fig. 1). The $q_{0}-z_{c}$ contours at $3 \sigma$ restrict the possible values of the transition redshift approximately between 0.5 and 1.0,

\footnotetext{
${ }^{4}$ The parameter $z_{t}$ is obtained solving the expression $0=0.5+\left(q_{0}-\right.$ $0.5)\left(z_{t}+1\right) e^{z_{c}^{2}-\left(z_{t}+z_{c}\right)^{2}}$.
} 
Table 1 Mean values for the model parameters $\left(h, q_{0}, z_{c}\right)$ derived from OHD and SN Ia measurements

\begin{tabular}{|c|c|c|c|c|c|c|c|c|}
\hline Data set & $\chi_{\min }^{2}$ & $h$ & $q_{0}$ & $z_{c}$ & $M_{b}^{1}$ & $\delta_{M}$ & $\alpha$ & $\beta$ \\
\hline $\mathrm{OHD}(\mathrm{CC})$ & 15 & $0.726_{-0.015}^{+0.015}$ & $-0.79_{-0.14}^{+0.20}$ & $0.81_{-0.26}^{+0.22}$ & - & - & - & - \\
\hline SNIa (JLA) & 683 & $0.722_{-0.18}^{+0.19}$ & $-0.44_{-0.15}^{+0.12}$ & $0.51_{-0.30}^{+0.40}$ & $-18.98_{-0.64}^{+0.50}$ & $-0.07_{-0.02}^{+0.02}$ & $0.14_{-0.006}^{+0.006}$ & $3.10_{-0.08}^{+0.08}$ \\
\hline SNIa (Pantheon) & 1035 & $0.50_{-0.34}^{+0.34}$ & $-0.54_{-0.14}^{+0.12}$ & $0.75_{-0.28}^{+0.32}$ & - & - & - & - \\
\hline Joint (CC+JLA) & 700 & $0.713_{-0.01}^{+0.01}$ & $-0.49_{-0.12}^{+0.11}$ & $0.51_{-0.20}^{+0.22}$ & $-19.01_{-0.04}^{+0.04}$ & $-0.07_{-0.02}^{+0.02}$ & $0.14_{-0.006}^{+0.006}$ & $3.11_{-0.08}^{+0.08}$ \\
\hline Joint (CC+Pantheon) & 1054 & $0.710_{-0.01}^{+0.01}$ & $-0.51_{-0.10}^{+0.09}$ & $0.61_{-0.18}^{+0.21}$ & - & - & - & - \\
\hline
\end{tabular}

Fig. 2 1D marginalized posterior distributions and the 2D $68 \%, 95 \%$, and $99.7 \%$ confidence levels for the $h, q_{0}$ and $z_{c}$ parameters for the joint analysis of SNIa+CC. The circle and the star represent the parameter mean values for the $\mathrm{CC}+\mathrm{JLA}$ and the $\mathrm{CC}+$ Pantheon samples, respectively

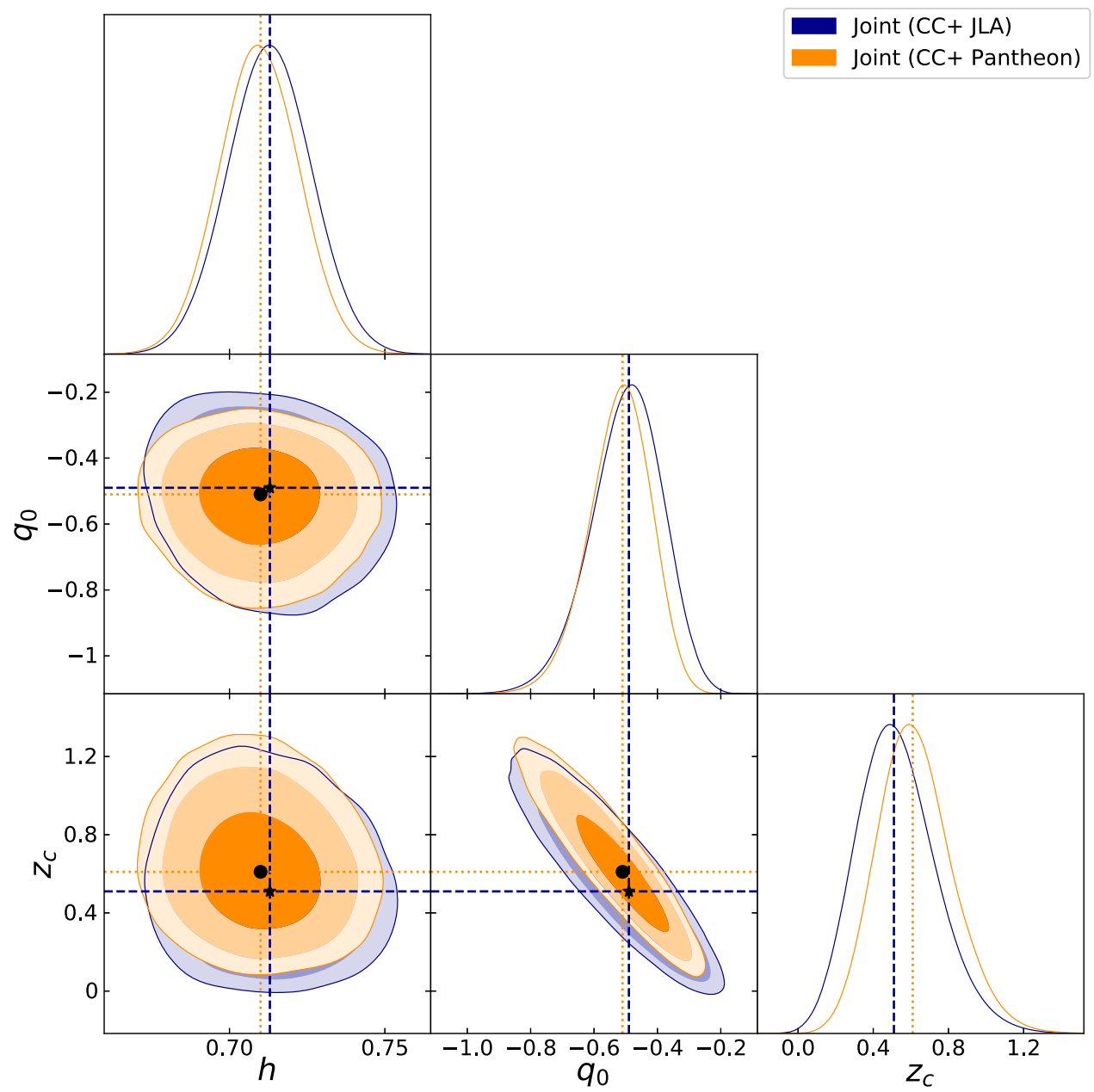

reproducing an accelerated cosmic phase at late times. Additionally, notice that the principal axes of the $h-q_{0}$ and $h$ $z_{c}$ confidence contours are parallel to the coordinates axes, indicating that these parameter pairs are uncorrelated. For the case of a spatially flat universe filled with a barotropic fluid (or more than one), it has been shown that $q_{0}$ only depends on the density parameters, and the corresponding EoS of the fluid $[18,19,21,22,52,53]$. This characteristic is also observed in our $h-q_{0}$ constraints, which further supports the proposed functional form for the deceleration parameter and the assumption of a barotropic fluid. A similar analysis can be obtained for the transition redshift $z_{t}$ (related to the $z_{c}$ parameter) in the sense that such parameter is associated with the density parameters of the Universe's components. The result for the $h-z_{c}$ constraints depicted in Fig. 2 shows also a lack of correlation between both parameters, reinforcing the proposed model in this work.

It is worth to note that the joint confidence contours using the Pantheon sample are slightly narrower than those obtained with the JLA sample. This feature is also present in the 1D histograms, those estimated with the Pantheon sample are slightly more tight (see also errors in Table 1). This is related to several systematic uncertainties in the measurements of the SNIa (e.g., photometry, and astrometry calibration, SN modeling, Milky Way extinction model), see [50]. Our results are consistent with those of [50], i.e. Pantheon 


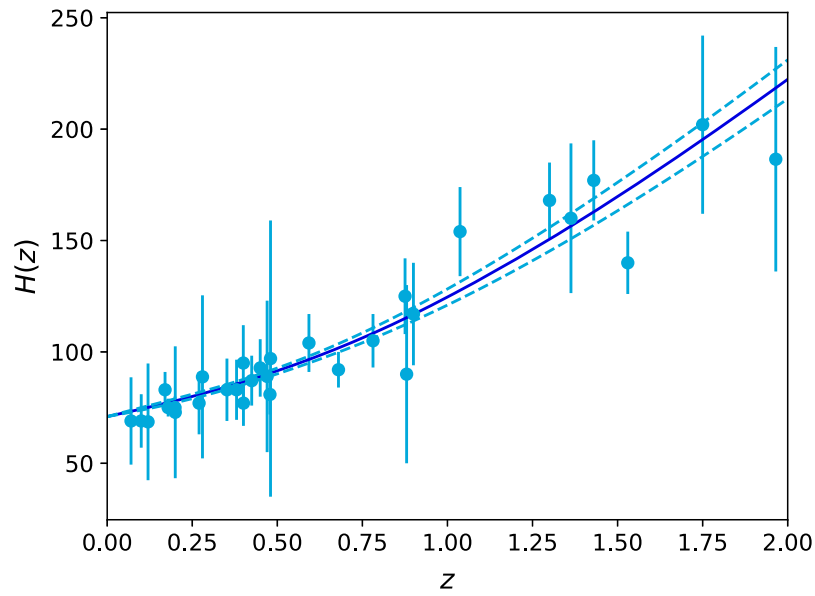

Fig. 3 Fit to OHD (left panel) and the reconstructed $q(z)$ (right panel) using the joint analysis (CC+Pantheon) constraints. The dashed and red shadow regions show the $1 \sigma$ confidence limits estimated from a

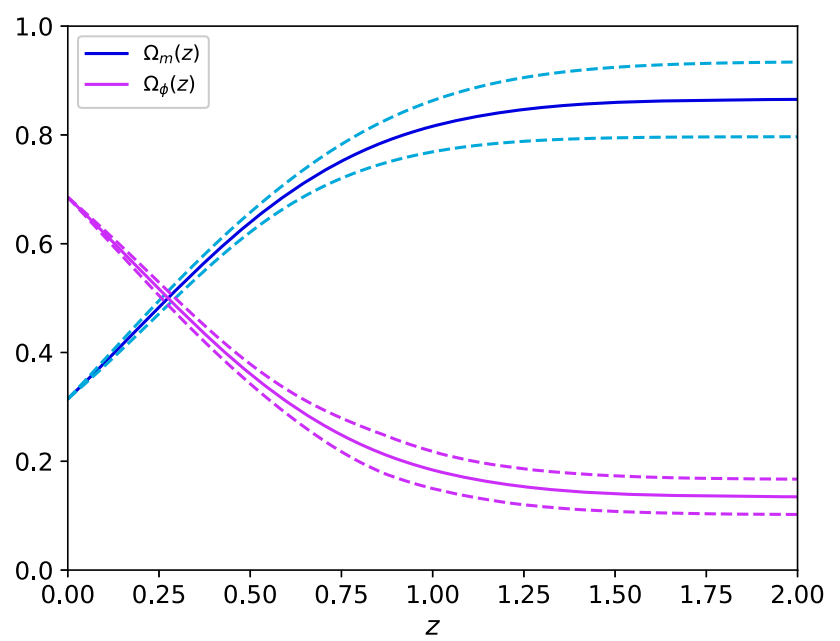

Fig. 4 The matter and DE density parameter of the Universe using the joint analysis mean values. The dashed regions show the $1 \sigma$ confidence limits estimated from a MCMC analysis

sample seems to provide tighter cosmological constraints than the JLA sample, although the difference is not statistically significant.

A numerical analysis of the roots of $q(z)$ allows to estimate the value of the transition redshift, $z_{t}=0.65_{-0.17}^{+0.19}$, for the joint data set using the Pantheon sample (we will only make use the results of this joint analysis hereinafter). This result is consistent with values reported in literature $[25,36,39,54-58]$, indicating that the Universe passed from a decelerated phase to an accelerated one at $z \approx 0.7$. The right panel of Fig. 3 illustrates the reconstructed $q(z)$ for the joint analysis constraints. Note that $q_{0}=-0.51_{-0.10}^{+0.09}$, and the reconstructed $\Omega_{m}(\mathrm{z})$ are in agreement with the dynamics of the standard cosmological model, as well with $[18,22,59$

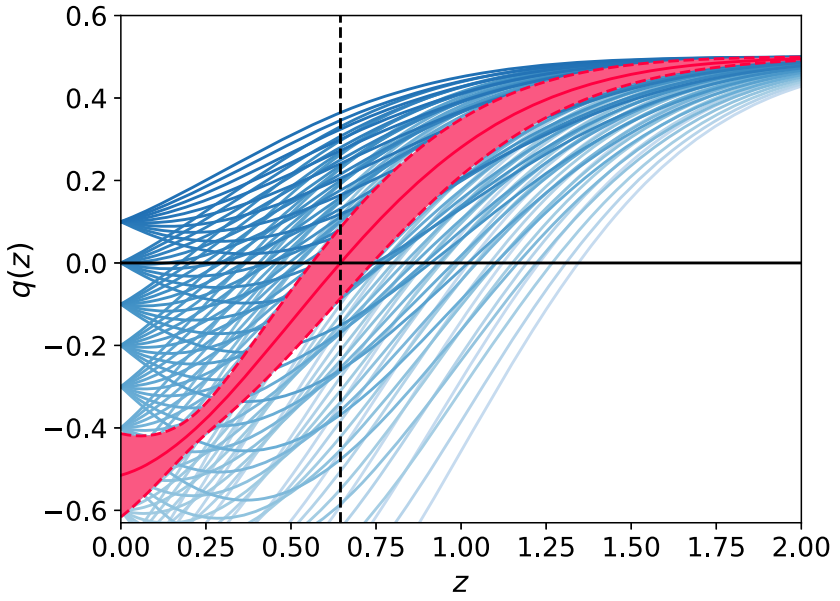

MCMC analysis. The black dashed line in the right panel represents the transitional redshift, $z_{t}$, for the joint analysis mean value

63]. The matter component is dominant with respect to the dark energy component for high redshift values, the opposite occurs at late times (see Fig. 4).

\section{Dynamical dark energy}

\subsection{The resulting EoS}

The left panel in Fig. 5 presents the EoS constructed from the Eq. (10) using the parameter mean values and $\Omega_{m, 0}=0.31$ [64]

$\omega(z)=\mathcal{A}(z) \times \frac{1}{1-\mathcal{B}(z)}$.

where $\mathcal{A}(z)$ is a function of $z$, and $\mathcal{B}(z)$ could be expressed as

$$
\begin{aligned}
\mathcal{B}(z)= & 0.31(1+z)^{0.5} \\
& \times e^{\left(-2 \xi\left(\operatorname{erf}\left(z+0.50_{-0.19}^{+0.20}\right)-\operatorname{erf}\left(0.50_{-0.19}^{+0.20}\right)\right)\right)} .
\end{aligned}
$$

Although Eq. (21) is a well-behaved function, from Eq. (22) is clear that the denominator may be zero, leading to a singularity in the EoS (see next section). A way to overcome this problem is studying the derivative of the EoS [23]. From Eq. (9), it is straightforward to show that

$$
\begin{aligned}
\frac{\mathrm{d} \omega(z)}{\mathrm{dz}}= & \frac{2}{3}\left(1-\Omega_{m}(z)\right)\left(q ( z ) \left(\frac{1}{z+1}\right.\right. \\
& \left.\left.-2\left(z+z_{c}\right)\right) q_{1}\left(2\left(z+z_{c}\right)-\frac{1}{1+z}\right)\right) \\
& +3\left(q(z)-\frac{1}{2}\right) \Omega_{m}(z)\left((1+z)\left(1-\Omega_{m}(z)\right)^{2}\right)^{-1} .
\end{aligned}
$$




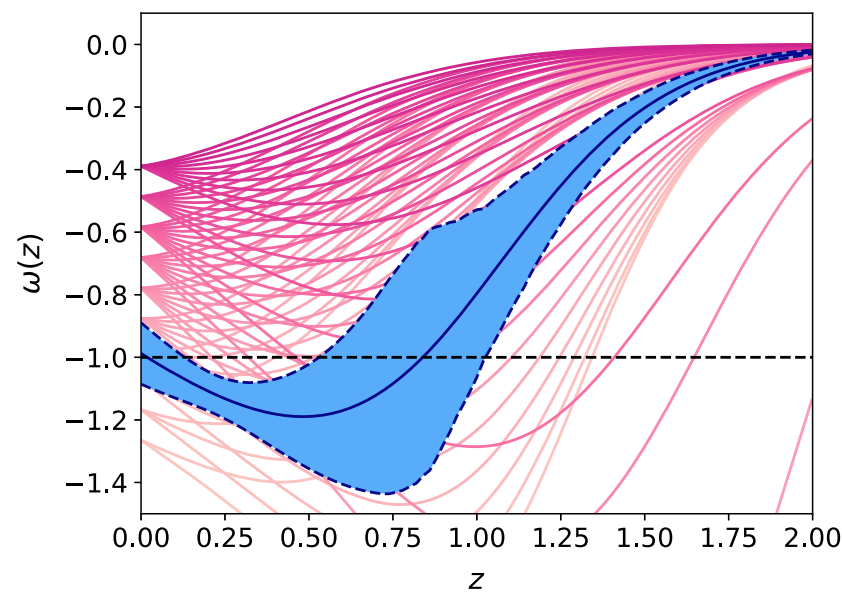

Fig. 5 The $\omega$ reconstruction using the joint analysis $(\mathrm{CC}+\mathrm{Pantheon})$ mean values and its functional form (left panel). The horizontal black dashed line represent the phantom divide. The right panel shows the

The equation $\omega$ and the derivative $d \omega / d z$ are shown in the right panel of Fig. 5. The value of the EoS today, $\left.\omega(z)\right|_{z=0} \equiv$ $\omega_{0}=-0.99_{-0.1}^{+0.1}$ is consistent with the standard cosmological model i.e. with the cosmological constant. Note that around $z \approx 1$ the EoS changes concavity (inflexion point), producing a maximum in $d \omega / d z$. Furthermore, the first derivative of $\omega$ with respect $z$ gives a value, as shown in Fig. 5, of $d \omega /\left.d z\right|_{z=0}=-0.97_{-0.37}^{+0.37}$, consistent with [54].

\subsection{Discriminating dark energy models}

The nature of DE is connected to the characteristics of the EoS. The reconstruction of the EoS by Eq. (9) may have singular points on its domain, i.e. it might diverge, which occurs when the denominator is equal to zero. To find the singular points we consider the next equation:

$1-\Omega_{m, 0}(1+z)^{3}\left(\frac{H_{0}}{H(z)}\right)^{2}=0$,

which can be written as (see A):

$1-\Omega_{m}(z)=0$.

We expect $\Omega_{m}(z)$ to be a monotonically increasing function from the present (at $z=0$ ), to a matter dominated epoch when $q(z) \rightarrow q_{1}=1 / 2$ (see Appendix A). As Eq. (3) asymptotically tends to $q(z) \sim q_{1}$ as $z \rightarrow \infty$ [65], our EoS reconstruction is valid only from today to an epoch of the Universe when matter dominates. In future works, we expect to study a more general parameterization of the deceleration by using $q_{1}$ as free parameter.

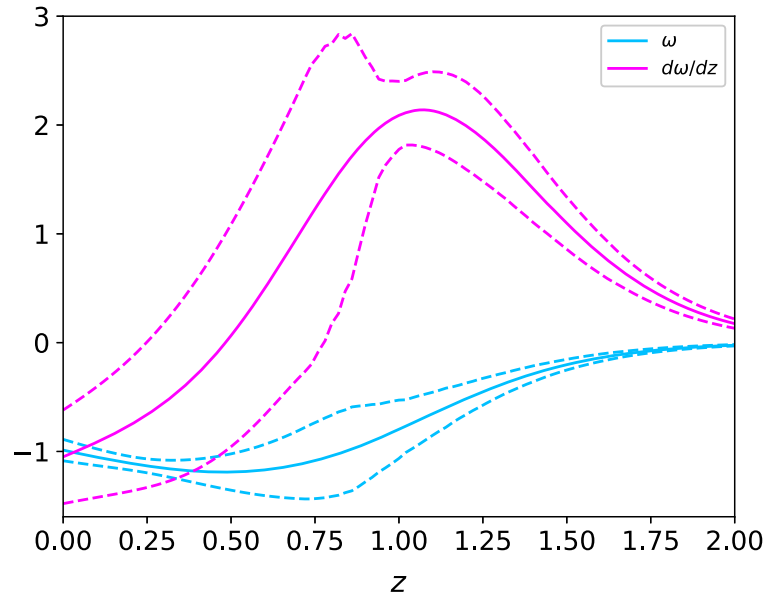

effective EoS (cyan) and its derivative with respect to $z$ (magenta). The regions delimited by dashed lines show the $1 \sigma$ confidence limits estimated from a MCMC analysis

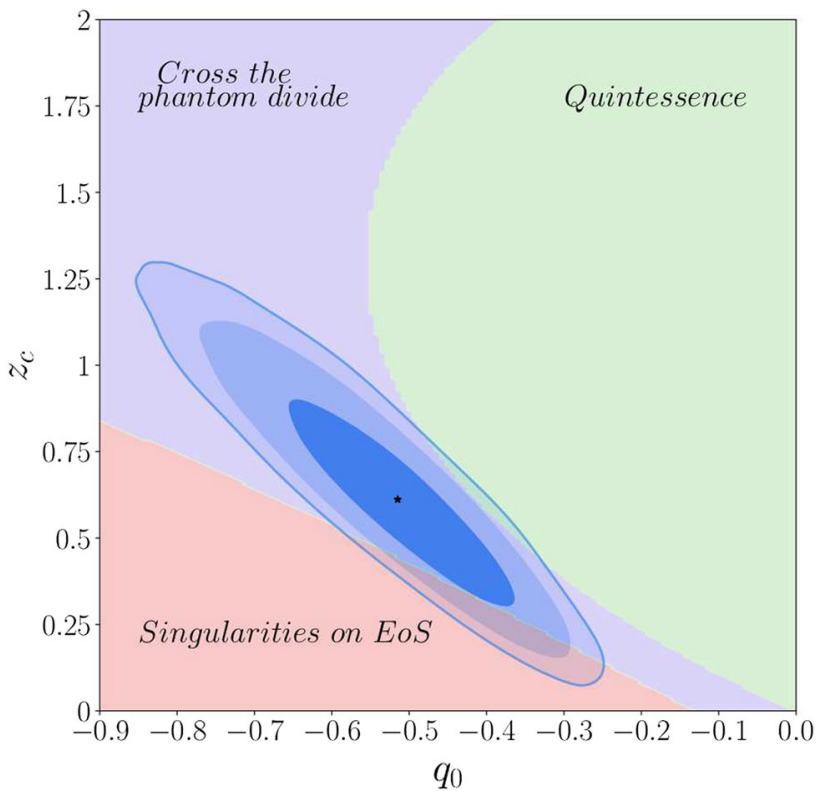

Fig. 6 Decision regions in the parameter space and the 68\%, 95\%, $99.7 \%$ confidence levels for the $q_{0}$ and $z_{c}$ parameters for the joint analysis constraints. The classification of the EoS (depending on the given $\omega$ value) is represented in different regions: green for quintesence models; purple for quintom models; red for EoS with singular points. The black star represents the joint analysis (CC + Pantheon) mean values for $z_{c}$ and $q_{0}$

The condition given by Eq. (25) is satisfied for $z>0$. As comment before, the EoS is valid too in a matter dominated epoch, i.e., $z>>1$, let us assume for simplicity that $z \rightarrow \infty$. Thus, by substituting the Eq. (4) into Eq. (29), the limit for $\Omega_{m}(z)$ at such epoch is:

$\lim _{z \rightarrow \infty} \Omega_{m}(z)=\Omega_{m, 0} \exp \left[2 \xi\left(\operatorname{erf}\left(z_{c}\right)-1\right)\right]$. 
Considering that the reconstruction of $\Omega_{m}(z)$ for our model is a monotonic increasing function for $z \geq 0$ (see Appendix A), for given a pair of fixed $q_{0}$ and $z_{c}$ there exist a real positive value of the redshift $z$ for which Eq. (9) will contain a singular point if

$\Omega_{m, 0} \exp \left[2 \xi\left(\operatorname{erf}\left(z_{c}\right)-1\right)\right]>1$.

Figure 6 illustrates the $q_{0}-z_{c}$ region bounded for this inequality, showing two regions of interest: the quintessence region and, where the EoS crosses the phantom divide. In the case that $\omega(z) \in[-1,1]$, the bartropic fluid can be represented with a minimally coupled scalar field, known as quintessence DE model and which is consistent with $\Lambda \mathrm{CDM}$ [66], but if $\omega(z)<-1$ the behavior of the fluid is represented as a phantom DE [4]. Since in our proposed EoS (see Eq. 9) does not exist an evident restriction for its codomain, it is important to know whether the reconstruction go through the phantom divide, defined as $\omega=-1$. If the EoS cross the phantom divide, the DE behavior can be represented by the dynamics of more than a single scalar field [67], e.g. a combination of a negative-kinetic and a normal scalar field, as quintom DE [68]. Notice that our joint analysis mean values for $q_{0}$ and $z_{c}$ rely on both regions, panthom and quintessence.

Quintessence models can be classified by the behavior of the potential associated with the scalar field. The two categories are thawing models and freezing (tracking) models (see $[17,69]$ and references therein). In the thawing models, the scalar field is frozen at early times due to the Hubble parameter damping, ${ }^{5}$ while at late times the friction term becomes subdominant. The $\omega(z)$ is a decreasing function that asymptotically reaches the cosmological constant EoS (i.e. $\omega \approx-1$ ) at early times. In the freezing models, the scalar potential is steep enough at early times to develop the kinetic term, while at late times it becomes shallower allowing the slowing down of the scalar field. The $\omega(z)$ is an increasing function that tends to the Cosmological Constant EoS at late times. An effective tool to discriminate between these models is the $\omega^{\prime}-\omega$ plane, where $\omega^{\prime}=d \omega / d \ln a$ [70]; since different models are bounded by different regions [70-72].

A phantom DE can be represented by a scalar field minimally coupled to gravity with a non-canonical negativekinetic energy term, and whose energy density grows with time. Thus, the tracking behavior of a phantom model can be depicted in the $\omega^{\prime}-\omega$ plane [71]. Because in the quintom models the evolution equations of the negative-kinetic and the normal scalar fields are independent [12], the potential behavior can be classified by the quintessence and phantom discrimination regions obtained separately. Figure 7 shows the discrimination regions for quintessence (thaw-

\footnotetext{
5 Indicates that the dynamics of the scalar field is governed by the Klein-Gordon equation.
}

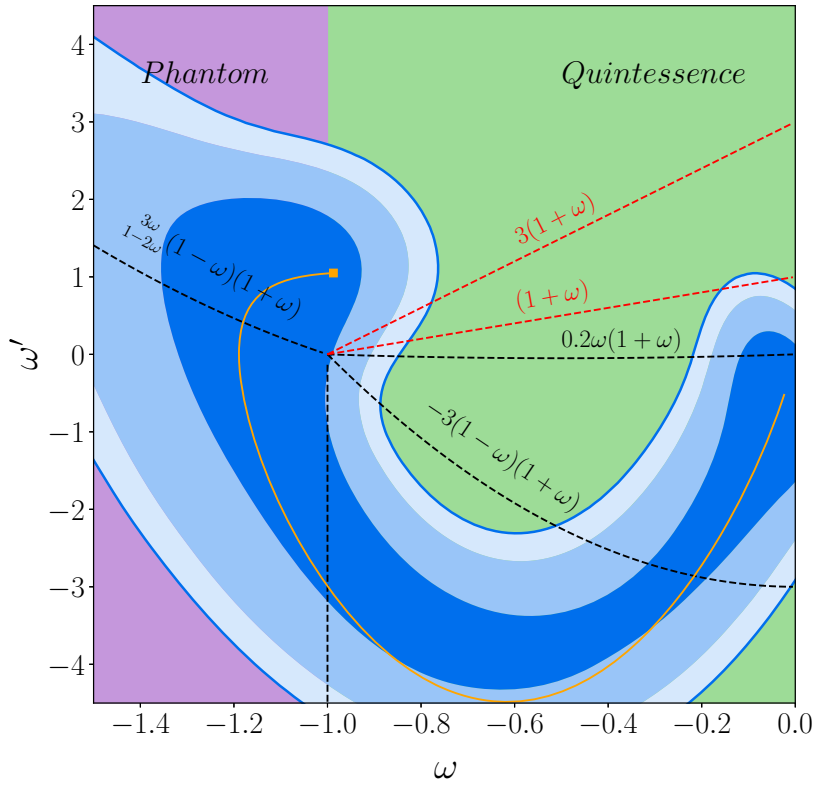

Fig. 7 Discrimination regions for quintessence (thawing and freezing behavior) and phantom models in the $\omega^{\prime}-\omega$ plane. The red dashed lines represent the bounds for the thawing discrimination region, where $\omega^{\prime}=$ $3(1+\omega)$ is the upper bound and $\omega^{\prime}=(1+\omega)$ is the lower bound [70]. The black dashed lines in the quintessence region $(\omega>-1)$ are the bounds for freezing models, where $\omega^{\prime}=0.2 \omega(1+\omega)$ is the upper bound, and $\omega^{\prime}=-3(1-\omega)(1+\omega)$ is the lower bound, see [72]. In the phantom region $(\omega<-1), \omega^{\prime}=3 \omega(1-\omega)(1+\omega) /(1-2 \omega)$ is the upper bound, and $\omega=-1$ is the lower bound, see [71]. In shades of blue are the $68 \%, 95 \%, 99.7 \%$ confidence levels for the reconstruction of $\omega$ and $\omega^{\prime}$ using the joint constraints (CC++Pantheon), and the orange line is the mean value of these reconstructions. The orange square is the value at redshift $z=0$

ing and freezing behavior) and phantom models in the $\omega^{\prime}-$ $\omega$ plane. The thawing discrimination region is delimited between $\omega^{\prime}=1+\omega$ (lower bound) and $\omega^{\prime}=3(1+\omega)$ (upper bound) [70]. The freezing quintessence limits are provided by $\omega^{\prime}=0.2 \omega(1+\omega)$ (upper bound) and $\omega^{\prime}=-3(1-\omega)(1+\omega)$ (lower bound) $[71,72]$. The upper bound for phantom region is $\omega^{\prime}=3 \omega(1-\omega)(1+\omega) /(1-2 \omega)$ [71]. As shown in Fig. 7, our analysis exclude thawing behaviour of the scalar field, being consistent with [73]. Notice that our joint constraints on the $q(z)$ parameters crosses both the quintessence and phantom regions, hence, confirming that our results are consistent with DE models that crosses the phantom divide, e.g. quintom DE.

\section{Summary}

There are several ways to approach the dynamical evolution of the Universe with the aim of describing the late and early epoch expansion. Many models of DE, such as canonical and negative-kinetic scalar field models, are represented by a barotropic fluid. Recent observations indicate a transition 
between a decelerated and an accelerated phase of the cosmic expansion, from a matter dominated epoch to recent times, respectively. In this work we proposed a new phenomenological parameterization of the deceleration parameter, Eq. (3), to approach the accelerated evolution of the cosmic expansion. The proposed form of $q(z)$ is a well behaved equation that can represent a step-like transition for this parameter, as well as being suitable for an analytical reconstruction of the Hubble parameter and the DE EoS. The behaviour of this new $q(z)$ parameterization allows to constrain minimally coupled scalar field DE models, as well as models which the DE EoS crosses the phantom divide. For minimally coupled scalar field models, as quintessence, the changes in the concavity of the proposed $q(z)$ points to a more general fitting of the dynamics of the scalar field, as thawing and freezing behaviours.

We performed an MCMC Bayesian analysis to constrain the $q(z)$ parameters using the OHD, and two SNIa samples: the JLA, and Pantheon. For the join analysis (CC + Pantheon) we obtain $q_{0}=-0.51_{-0.10}^{+0.09}, h=0.710_{-0.01}^{+0.01}$, and $z_{t}=0.65_{-0.17}^{+0.19}$, which are consistent with values reported by other authors. The reconstruction of the EoS (see Fig. 6) using these values crosses the phantom divide, rejecting the quintessence DE models. Our result points to a quintom DE, and it is consistent with a non parametric reconstruction of the EoS using the latest cosmological observations (see Ref. [74]) within the range of validity of the Eq. (9).

The behavior of the two free-parameter reconstruction of the EoS (Eq. 10) is a more general expression, including both the thawing or freezing scalar field models. Indeed, the functional form of $\omega$ does not impose an apriori category of scalar field model for its entire domain. Furthermore, the discrimination analysis we presented in Fig. 7 is also consistent with a quintom DE model. Quintom DE is only an example of a model that need the dynamics of more than a single scalar field to cross the phantom divide. Considering another set of models would imply that the energy-momentum tensor may deviate from the perfect-fluid form as those studied by [75], which are related to Hordenski gravity [76], and consistent with the recent GW observations [77]. Then we may assume that these models should be non significant deviation from the perfect-fluid form in order to remain the validity of Eq. (9). Another possibility is to invoke non-linear physics to explain the transition of the phantom divide with a single scalar field, as mentioned in [67]. The confidence contours for $\omega^{\prime}$ vs. $\omega$, are not subsets of a single model region within the regions delimited by thawing and freezing models. This is a complex behavior of our two free parameter reconstruction of the EoS, in contrast to the parameterizations analyzed in Ref. [17]

In a future work, we plan to extend the study presented here, and analyze the consequences of the cosmic expansion in a early epoch by setting $q_{1}$ as a free parameter, and its repercussions on the behavior of the effective EoS. Also to consider a more general set of imperfect DE models.

Acknowledgements The authors thank the anonymous referee for invaluable remarks and suggestions, that helped to improve the paper. The authors thank Luis Ureña for his thoughtful comments. This research has been carried out thanks to PROGRAMA UNAM-DGAPAPAPIIT IA102517. J.M. acknowledges support from Grant 3160674 (CONICYT/FONDECYT), and thanks the hospitality of the staff of IA-Ensenada where part of this work was done.

Data Availability Statement This manuscript has no associated data or the data will not be deposited. [Authors' comment: This is theoretical research work, and is based upon the analysis of public observational data (previously published by different authors). So no additional data are associated with this work.]

Open Access This article is distributed under the terms of the Creative Commons Attribution 4.0 International License (http://creativecomm ons.org/licenses/by/4.0/), which permits unrestricted use, distribution, and reproduction in any medium, provided you give appropriate credit to the original author(s) and the source, provide a link to the Creative Commons license, and indicate if changes were made.

Funded by SCOAP $^{3}$.

\section{A The behavior of $\Omega_{m}(z)$ and the singularities of $\omega$}

By considering the definition of the matter density in terms of $z$ :

$\Omega_{m}(z)=\frac{\rho_{m}(z)}{3 H^{2}(s)}$,

where $\rho_{m}(z)=3 H_{0}^{2} \Omega_{m, 0}(1+z)^{3}$, Eq. (28) can be rewritten as

$\Omega_{m}(z)=\Omega_{m, 0}(1+z)^{3}\left(\frac{H_{0}}{H(z)}\right)^{2}$.

Let us calculate the first derivative of $\Omega_{m}(z)$ with respect of $z$

$$
\begin{aligned}
\frac{d \Omega_{m}(z)}{d z}= & 3 \Omega_{m, 0}\left(\frac{H_{0}}{H(z)}\right)^{2}(1+z)^{2} \\
& -2 \Omega_{m, 0} \frac{H_{0}^{2}}{H(z)^{3}} \frac{d H(z)}{d z}(1+z)^{3},
\end{aligned}
$$

from Eq. (2) $\frac{d H(z)}{d z}=H(z) \frac{1+q(z)}{1+z}$, simplifying Eq. (30)

$\frac{d \Omega_{m}(z)}{d z}=\Omega_{m, 0}(1+z)^{2}\left(\frac{H_{0}}{H(z)}\right)^{2}(1-2 q(z))$,

By the reconstruction of the Hubble parameter using the joint dataset, $H(z)>0$ and $q(z)<1 / 2$ for $z \geq 0$, see Fig. 3 . Introducing both considerations in Eq. (31), we obtain

$\frac{d \Omega_{m}(z)}{d z}>0 \quad \forall z \geq 0$, 
for our model. Thus, in this case, $\Omega_{m}(z)$ is a monotonic increasing function for all $z \geq 0$. Given Eq. (26) and $\Omega_{m}(0)=\Omega_{m, 0}<1$ [54], then the codomain of this function is delimited:

$$
\begin{aligned}
& \Omega_{m}(z) \in\left[\Omega_{m, 0}, \Omega_{m, 0} \exp \left(2 \xi\left(\operatorname{erf}\left(z_{c}\right)-1\right)\right)\right) \\
& \forall z \geq 0 .
\end{aligned}
$$

Let us consider the next cases:

$$
\begin{aligned}
& \text { - If } \Omega_{m, 0} \exp \left(2 \xi\left(\operatorname{erf}\left(z_{c}\right)-1\right)\right)<1: \\
& \quad \Rightarrow \Omega_{m}(z)<1 \quad \forall z \geq 0 \\
& \Rightarrow 1-\Omega_{m}(z)>0 \forall z \geq 0
\end{aligned}
$$

then, there is not a value of $z \geq 0$ such that $\omega_{\text {eff }}(z)$ diverges.

- If $\Omega_{m, 0} \exp \left(2 \xi\left(\operatorname{erf}\left(z_{c}\right)-1\right)\right)=1$ :

$$
\begin{aligned}
& \Rightarrow \Omega_{m}(z) \sim 1 \quad \text { as } z \rightarrow \infty \\
& \Rightarrow \omega_{\mathrm{eff}}(z) \rightarrow \infty \text { as } z \rightarrow \infty
\end{aligned}
$$

then, $\omega_{\text {eff }}(z)$ diverges as $z \rightarrow \infty$.

- If $\Omega_{m, 0} \exp \left(2 \xi\left(\operatorname{erf}\left(z_{c}\right)-1\right)\right)>1$ :

Because the codomain of $\Omega_{m}(z)$ is delimited as Eq. (33),

$$
1 \in\left[\Omega_{m, 0}, \Omega_{m, 0} \exp \left(2 \xi\left(\operatorname{erf}\left(z_{c}\right)-1\right)\right)\right),
$$

then there is a value $z^{\prime}>0$ such that $\Omega_{m}\left(z^{\prime}\right)=1$

$$
\Rightarrow 1-\Omega_{m}\left(z^{\prime}\right)=0 \quad \text { where } z^{\prime}>0 .
$$

Therefore, the last case gives the condition to have a singular point of $\omega_{\text {eff }}$.

\section{References}

1. Planck Collaboration, P.A.R. Ade, N. Aghanim, M. Arnaud, M. Ashdown, J. Aumont, C. Baccigalupi, A.J. Banday, R.B. Barreiro, J.G. Bartlett, et al. Planck 2015 results. XIII. Cosmological parameters. Astron. Astrophys. 594, A13 (2016)

2. A.G. Riess, A.V. Filippenko, P. Challis, A. Clocchiatti, A. Diercks et al., Observational evidence from supernovae for an accelerating universe and a cosmological constant. Astron. J. 116(3), 1009 (1998)

3. S. Perlmutter, G. Aldering, G. Goldhaber, R.A. Knop, P. Nugent, others, and The Supernova Cosmology Project. Measurements of and from 42 high-redshift supernovae. Astrophys. J. 517(2), 565 (1999)

4. E.J. Copeland, M. Sami, S. Tsujikawa, Dynamics of dark energy. Int. J. Mod. Phys. D 15, 1753-1936 (2006)

5. K. Bamba, S. Capozziello, S. Nojiri, S.D. Odintsov, Dark energy cosmology: the equivalent description via different theoretical models and cosmography tests. Astrophys. Sp. Sci. 342, 155-228 (2012)

6. M. Li, X.-D. Li, S. Wang, Y. Wang, Dark energy. Commun. Theor. Phys. 56, 525-604 (2011)
7. C. Wetterich, Cosmology and the fate of dilatation symmetry. Nucl. Phys. B 302, 668-696 (1988)

8. P.J.E. Peebles, B. Ratra, Cosmology with a time variable cosmological constant. Astrophys. J. 325, L17 (1988)

9. R.R. Caldwell, R. Dave, P.J. Steinhardt, Cosmological imprint of an energy component with general equation of state. Phys. Rev. Lett. 80, 1582-1585 (1998)

10. R.R. Caldwell, A phantom menace? Phys. Lett. B 545, 23-29 (2002)

11. T. Chiba, T. Okabe, M. Yamaguchi, Kinetically driven quintessence. Phys. Rev. D 62, 023511 (2000)

12. Z.-K. Guo, Y.-S. Piao, X.-M. Zhang, Y.-Z. Zhang, Cosmological evolution of a quintom model of dark energy. Phys. Lett. B 608, 177-182 (2005)

13. C. Armendariz-Picon, V.F. Mukhanov, P.J. Steinhardt, A dynamical solution to the problem of a small cosmological constant and late time cosmic acceleration. Phys. Rev. Lett. 85, 4438-4441 (2000)

14. C. Armendariz-Picon, V.F. Mukhanov, P.J. Steinhardt, Essentials of k essence. Phys. Rev. D 63, 103510 (2001)

15. M. Chevallier, D. Polarski, Accelerating universes with scaling dark matter. Int. J. Mod. Phys. D 10, 213-224 (2001)

16. E.V. Linder, Exploring the expansion history of the universe. Phys. Rev. Lett. 90, 091301 (2003)

17. G. Pantazis, S. Nesseris, L. Perivolaropoulos, Comparison of thawing and freezing dark energy parametrizations. Phys. Rev. D 93(10), 103503 (2016)

18. O. Luongo, Cosmography with the hubble parameter. Mod. Phys. Lett. A 26(20), 1459-1466 (2011)

19. A. Aviles, C. Gruber, O. Luongo, H. Quevedo, Cosmography and constraints on the equation of state of the Universe in various parametrizations. Phys. Rev. D 86(12), 123516 (2012)

20. C. Gruber, O. Luongo, Cosmographic analysis of the equation of state of the universe through padé approximations. Phys. Rev. D 89, 103506 (2014)

21. M. Demianski, E. Piedipalumbo, C. Rubano, P. Scudellaro, Highredshift cosmography: new results and implications for dark energy. Mon. Not. R. Astron. Soc. 426(2), 1396-1415 (2012)

22. C. Gruber, O. Luongo, Cosmographic analysis of the equation of state of the universe through padé approximations. Phys. Rev. D 89(10), 103506 (2014)

23. M.-J. Zhang, H. Li, J.-Q. Xia, What do we know about cosmography. Eur. Phys. J. C 77, 434 (2017)

24. A.R. Sandage, Cosmology: a search for two numbers. Phys. Today 23, 34-41 (1970)

25. A.A. Mamon, S. Das, A divergence free parametrization of deceleration parameter for scalar field dark energy. Int. J. Mod. Phys. D 25(03), 1650032 (2016)

26. S. del Campo, I. Duran, R. Herrera, D. Pavon, Three thermodynamically-based parameterizations of the deceleration parameter. Phys. Rev. D 86, 083509 (2012)

27. R. Nair, S. Jhingan, D. Jain, Cosmokinetics: a joint analysis of standard candles, rulers and cosmic clocks. JCAP 1201, 018 (2012)

28. B. Santos, J.C. Carvalho, J.S. Alcaniz, Current constraints on the epoch of cosmic acceleration. Astropart. Phys. 35, 17-20 (2011)

29. A.A. Mamon, S. Das, A divergence-free parametrization of deceleration parameter for scalar field dark energy. International Journal of Modern Physics D 25(03), 1650032 (2016)

30. A.A. Mamon, S. Das, A parametric reconstruction of the deceleration parameter. Eur. Phys. J. C 77(7), 495 (2017)

31. Bruce A Bassett, Pier Stefano Corasaniti, and Martin Kunz. The essence of quintessence and the cost of compression. The Astrophysical Journal Letters, 617(1):L1, (2004)

32. M.S. Turner, A.G. Riess, Do SNe Ia provide direct evidence for past deceleration of the universe? Astrophys. J. 569, 18 (2002) 
33. J.E. Bautista et al., Measurement of baryon acoustic oscillation correlations at $z=2.3$ with SDSS DR12 Ly $\alpha$-forests. Astron. Astrophys. 603, A12 (2017)

34. A. Al Mamon, S. Saha, Constraints on a generalized deceleration parameter from cosmic chronometers and its thermodynamic implications (2017)

35. J.V. Cunha, J.A.S. Lima, Transition redshift: new kinematic constraints from supernovae. Mon. Not. R. Astron. Soc. 390(1), 210 217 (2008)

36. J.V. Cunha, Kinematic constraints to the transition redshift from SNe Ia union data. Phys. Rev. D 79, 047301 (2009)

37. Y. Gong, A. Wang, Observational constraints on the acceleration of the universe. Phys. Rev. D 73, 083506 (2006)

38. X. Lixin, L. Jianbo, Cosmic constraints on deceleration parameter wih Sne Ia and CMB. Mod. Phys. Lett. A 24(05), 369-376 (2009)

39. X. Lix-In, C.-W. Zhang, B.-R. Chang, H.-Y. Liu, Constraints to deceleration parameters by recent cosmic observations. Mod. Phys. Lett. A 23, 1939-1948 (2008)

40. H.K. Jassal, J.S. Bagla, T. Padmanabhan, WMAP constraints on low redshift evolution of dark energy. Mon. Not. R. Astron. Soc. 356, L11-L16 (2005)

41. C.-J. Feng, X.-Y. Shen, P. Li, X.-Z. Li, A new class of parametrization for dark energy without divergence. JCAP 1209, 023 (2012)

42. I. Sendra, R. Lazkoz, Supernova and baryon acoustic oscillation constraints on (new) polynomial dark energy parametrizations: current results and forecasts. Mon. Not. R. Astron. Soc. 422, 776-793 (2012)

43. M. Rezaei, M. Malekjani, S. Basilakos, A. Mehrabi, D.F. Mota, Constraints to dark energy using PADE parameterizations. Astrophys. J. 843(1), 65 (2017)

44. E.M. Barboza Jr., J.S. Alcaniz, A parametric model for dark energy. Phys. Lett. B 666, 415-419 (2008)

45. P.S. Corasaniti, E.J. Copeland, Model independent approach to the dark energy equation of state. Phys. Rev. D 67(6), 063521 (2003)

46. P.S. Corasaniti, M. Kunz, D. Parkinson, E.J. Copeland, B.A. Bassett, Foundations of observing dark energy dynamics with the Wilkinson microwave anisotropy probe. Phys. Rev. D 70(8), 083006 (2004)

47. R. Jimenez, A. Loeb, Constraining cosmological parameters based on relative galaxy ages. Astrophys. J. 573, 37-42 (2002)

48. J. Magana, M.H. Amante, M.A. Garcia-Aspeitia, V. Motta, The Cardassian expansion revisited: constraints from updated Hubble parameter measurements and Type Ia Supernovae data. Mon. Not. R. Astron. Soc. 476, 1036 (2018)

49. M. Betoule et al., Improved cosmological constraints from a joint analysis of the SDSS-II and SNLS supernova samples. Astron. Astrophys. 568, A22 (2014)

50. D.M. Scolnic et al., The Complete Light-curve Sample of Spectroscopically Confirmed SNe Ia from Pan-STARRS1 and Cosmological Constraints from the Combined Pantheon Sample. Astrophys. J. 859(2), 101 (2018)

51. D. Foreman-Mackey, D.W. Hogg, D. Lang, J. Goodman, emcee: the MCMC hammer. PASP 125, 306 (2013)

52. J.A.S. Lima, J.F. Jesus, R.C. Santos, M.S.S. Gill. Is the transition redshift a new cosmological number? (2012)

53. Yu. L. Bolotin, V. A. Cherkaskiy, O. A. Lemets, D. A. Yerokhin, and L. G. Zazunov. Cosmology In Terms Of The Deceleration Parameter. Part I. (2015)

54. A.G. Riess et al., Type Ia supernova discoveries at $\mathrm{z}>1$ from the hubble space telescope: evidence for past deceleration and constraints on dark energy evolution. Astrophys. J. 607, 665-687 (2004)

55. A.A. Mamon, S. Das, A parametric reconstruction of the deceleration parameter. Eur. Phys. J. C 77(7), 495 (2017)
56. N. Rani, D. Jain, S. Mahajan, A. Mukherjee, N. Pires, Transition redshift: new constraints from parametric and nonparametric methods. J. Cosmol. Astropart. Phys. 2015(12), 045 (2015)

57. E.E.O. Ishida, R.R.R. Reis, A.V. Toribio, I. Waga, When did cosmic acceleration start? How fast was the transition? Astropart. Phys. 28(6), 547-552 (2008)

58. M.V. dos Santos, R.R.R. Reis, I. Waga, Constraining the cosmic deceleration-acceleration transition with type Ia supernova, BAO/CMB and H (z) data. J. Cosmol. Astropart. Phys. 2016(02), 066 (2016)

59. A. Aviles, C. Gruber, O. Luongo, H. Quevedo, Cosmography and constraints on the equation of state of the universe in various parametrizations. Phys. Rev. D 86(12), 123516 (2012)

60. A. Aviles, J. Klapp, O. Luongo, Toward unbiased estimations of the statefinder parameters. Phys. Dark Univ. 17, 25-37 (2017)

61. R. Giostri, M. Vargas, I. dos Santos, R.R.R.R. Waga, M.O. Calvao, B.L. Lago, From cosmic deceleration to acceleration: new constraints from SN Ia and BAO/CMB. J. Cosmol. Astropart. Phys. 2012(03), 027 (2012)

62. S.D.P. Vitenti, M. Penna-Lima, A general reconstruction of the recent expansion history of the universe. J. Cosmol. Astropart. Phys. 2015(09), 045 (2015)

63. Z. Davari, M. Malekjani, M. Artymowski, New parametrization for unified dark matter and dark energy. Phys. Rev. D 97(12), 123525 (2018)

64. N. Aghanim et al., Planck 2018 results. VI, Cosmological parameters (2018)

65. N.G. De Bruijn, Asymptotic Methods in Analysis, vol. 4 (Courier Corporation, North Chelmsford, 1970)

66. Á. de la Cruz-Dombriz, P.K.S. Dunsby, O. Luongo, L. Reverberi, Model-independent limits and constraints on extended theories of gravity from cosmic reconstruction techniques. J. Cosmol. Astropart. Phys. 2016(12), 042 (2016)

67. A. Vikman, Can dark energy evolve to the phantom? Phys. Rev. D 71(2), 023515 (2005)

68. B. Feng, X. Wang, X. Zhang, Dark energy constraints from the cosmic age and supernova. Phys. Lett. B 607, 35-41 (2005)

69. A. Sangwan, A. Tripathi, H.K. Jassal, Observational constraints on quintessence models of dark energy (2018)

70. R.R. Caldwell, E.V. Linder, Limits of quintessence. Phys. Rev. Lett. 95(14), 141301 (2005)

71. T. Chiba, W and w' of scalar field models of dark energy. Phys. Rev. D 73, 063501 (2006). [Erratum: Phys. Rev. D80, 129901 (2009)]

72. R.J. Scherrer, Dark energy models in the w-w' plane. Phys. Rev. D 73, 043502 (2006)

73. S. Dhawan, A. Goobar, E. Mrtsell, R. Amanullah, U. Feindt, Narrowing down the possible explanations of cosmic acceleration with geometric probes. JCAP 1707(07), 040 (2017)

74. G.-B.Zhao, M. Raveri, L. Pogosian, Y. Wang, R.G. Crittenden, W.J. Handley, W.J. Percival, F. Beutler, J. Brinkmann, C.-H. Chuang et al., Dynamical dark energy in light of the latest observations. Nat. Astron. 1(9), 627 (2017)

75. C. Deffayet, O. Pujolas, I. Sawicki, A. Vikman, Imperfect dark energy from kinetic gravity braiding. J. Cosmol. Astropart. Phys. 2010(10), 026 (2010)

76. G.W. Horndeski, Second-order scalar-tensor field equations in a four-dimensional space. Int. J. Theor. Phys. 10(6), 363-384 (1974)

77. P. Creminelli, F. Vernizzi, Dark energy after gw170817 and grb170817a. Phys. Rev. Lett. 119, 251302 (2017) 